\title{
विद्यार्थियों का व्यक्तित्व तथा संगीत सुनने की रूचि का अध्ययन
}

Leena Sharma ${ }^{1}$ and Dr. Mritunjay Sharma ${ }^{2}$

${ }^{1}$ M.Phil. Research Scholar, Department of Music, Himachal Pradesh University, Shimla ${ }^{2}$ Assistant Professor, Department of Music, Himachal Pradesh University, Shimla

\section{सार संक्षेपिका}

संगीत का मनुष्य के व्यक्तित्व पर गहरा प्रभाव पड़ता है। जो लोग संगीत की कुछ शैलियों को पसंद करते हैं वे विशिष्ट व्यक्तित्व लक्षणों को प्रदर्शित करते हैं। मानव की दिनचर्या में प्रयोग होने वाला पसंदीदा संगीत कहीं-न-कहीं उसके व्यक्तित्व को प्रभावित करता ही है इसमें कोई संदेह नहीं है। मनुष्य भिन्न-भिन्न प्रकार का संगीत सुनना पसंद करता है। संगीत की श्रेणी में शास्त्रीय संगीत, लोक संगीत, धार्मिक संगीत, पॉप, जैज़ इत्यादि आते हैं। लोगों के संगीत सुनने के प्रकारों से उनके व्यक्तित्व के विषय में जानकारी प्राप्त की जा सकती है या लोगों के व्यक्तित्व के बारे में जानकर उनके संगीत सुनने के प्रकारों के बारे में भी जानकारी उपलब्ध हो सकती है। हिमाचल प्रदेश विश्वविद्यालय, शिमला के विभिन्न स्नातकोत्तर विभागों में अध्ययनरत 50 छात्रों व 50 छात्राओं को शोध कार्य के लिए लिया गया। इन 100 विद्यार्थियों में से अन्तर्मुखी व्यक्तित्व के 49 विद्यार्थी व बहिर्मुखी व्यक्तित्व के 51 विद्यार्थी थे। इन 100 विद्यार्थियों में से 18 विद्यार्थियों ने गज़ल विधा को सर्वाधिक पंसद किया। दूसरे स्थान पर 17 विद्यार्थियों ने फिल्मी संगीत को पंसद किया। अन्तर्मुखी व्यक्तित्व के कुल 49 विद्यार्थियों में से 10 विद्यार्थियों ने शास्त्रीय संगीत, गज़ल व लोक संगीत को प्रथम स्थान पर चुना। दूसरे स्थान पर 8 विद्यार्थियों ने भजन विधा को चुना। बहिर्मुखी व्यक्तित्व के कुल 51 विद्यार्थियों में से 11 विद्यार्थियों ने फिल्मी संगीत विधा को प्रथम स्थान पर चुना तथा 10 विद्यार्थियों ने पाश्चात्य संगीत को दूसरे स्थान पर चुना। अतः यह कहा जा सकता है कि अन्तर्मुखी व बहिर्मुखी व्यक्तित्व के विद्यार्थियों ने भिन्न-भिन्न सांगीतिक विधाओं में अपनी रूचियाँ अलग अलग दर्शाई हैं।

बीज शब्द

संगीत, अन्तर्मुखी व बहिर्मुखी व्यक्तित्व, हिमाचल प्रदेश विश्वविद्यालय, रूचि

\section{ABSTRACT}

Music has a profound effect on the personality of a human being. People who like certain styles of music exhibit distinct personality traits. There is no doubt that the favorite music used in the routine of a human being affects his personality somewhere. Human beings like to listen to different types of music. The category of music includes classical music, folk music, religious music, pop, jazz etc. Information about their personality can be obtained from the types of music people listen to, or information about the types of people listening to their music can also be available. For this study, 50 male students and 50 female students studying in various postgraduate departments of Himachal Pradesh University, Shimla were selected. Out of these 100 students, there were 49 students of introverted personality and 51 students of extroverted personality. Out of these 100 students, 18 students liked Ghazal music the most. Second place was followed by 17 students who liked film music. Out of a total of 49 students of introverted personality, 10 students chose classical music, ghazal and folk music in the first place. In the second place, 8 students chose Bhajan music. Out of a total of 51 students of extroverted personality, 11 students chose film music in the first place and 10 students chose western music in the second place. Therefore, it can be said that students of introverted and extroverted personality have shown their interests in different musical disciplines.

Keywords

Music, Introvert and Extrovert Personality, himachal Pradesh University, Interest.

\section{भूमिका}

मनोविज्ञान और शिक्षा का ध्येय व्यक्ति के व्यक्तित्व को विकसित करना है। जब बच्चा जन्म लेता है तथा विकास की ओर अग्रसर होता है तो उसकी रुचियाँ चाहे वे किसी भी काम के लिए हों बदलती रहती हैं तथा उसके व्यक्तित्व को प्रभावित करती हैं। यदि किसी बालक के व्यक्तित्व, उसकी रूचि व शौक से अवगत हो जाएँ तो उसका भविष्य किस क्षेत्र में निर्मित करना है यह जानकारी ज्ञात की जा सकती है। 


\section{संगीत}

संगीत एक बहुआयामी कला है, जिसका मानव जीवन से गहरा सम्बन्ध है संगीत विधा सभी ललित कलाओं में शिखर पर है जो मानव व्यक्तित्व का पूर्ण रूप से विकास करती है। संगीत के कारण व्यक्ति का शारीरिक, मानसिक, सामाजिक, मनोवैज्ञानिक तथा आध्यात्मिक विकास सम्भव है। संगीतज्ञ अपनी रूचि के अनुसार अपने मनोभावों को स्वर, लय व ताल के माध्यम से प्रकट करता है तथा सकारात्मकता से अपने व दूसरों के जीवन में सुखों का संचार करता है।

\section{मनोविज्ञान}

मनोविज्ञान वह विषय है जो मनुष्य के व्यवहार का अध्ययन करता है, उसे इस बात से अवगत कराता है कि क्यों, कैसे व किन परिस्थितियों में वह और उसका व्यवहार दूसरे व्यक्तियों से बिल्कुल भिन्न है। मानव के व्यवहार को समझना एक कठिन कार्य है। मनोविज्ञान एक ऐसा कारक है जो मन व व्यवहार का बाहरी तथा आंतरिक दोनों तरह से अध्ययन करता है। सामान्य व्यक्तियों के व्यवहार और उनकी मानसिक प्रक्रियाओं से सम्बन्धित बातों का विश्लेषण करना सामान्य मनोविज्ञान है तथा असामान्य व्यक्ति जो मानसिक रूप से स्वस्थ नहीं है उनकी मनः स्थिति को जानना असामान्य मनोविज्ञान है।

रूचि

मानवीय व्यवहार का पथ प्रदर्शन करने और नियंत्रित करने में रूचि का प्रमुख योगदान है। रूचि वह प्रेरक शक्ति है जो उत्तेजित करती है स्थिर रखती है और नियमित बनाती है। रुचियों का शिक्षा में बहुत महत्व है क्योंकि शिक्षा के सभी कार्य इन पर निर्भर होते हैं, यदि अध्यापक विद्यार्थियों की आयु के अनुसार उनकी रुचियों के बारे में जानता है तो पाठ को अधिक रोचक प्रभावशाली तथा स्थायी बना सकता है परिणामस्वरूप विद्यार्थी के व्यक्तित्व का सर्वांगीण विकास होगा।

\section{व्यक्तित्व}

हम जो कुछ है और जो कुछ बनना चाहते हैं वह सब कुछ व्यक्तित्व के अंतर्गत आता है। व्यक्तित्व व्यक्ति की शारीरिक, बौद्धिक, भावात्मक, सामाजिक और चरित्रात्मक विशेषताओं का समूह है जो उसके दृष्टिकोणों, विश्वासों, इच्छाओं, आकांक्षाओं, व्यवहार, अनुभवों, आदतों, रूचियों भावनाओं तथा स्वभावगत विशेषताओं द्वारा प्रकट होता है। जनसाधारण की भाषा में व्यक्ति के बाहरी रूप को उसका व्यक्तित्व समझा जाता है अर्थात् व्यक्ति की वेश-भूषा, शक्ल-सूरत, क्रियाओं, आदतों आदि को उसका व्यक्तित्व समझ लिया जाता है। व्यक्तित्व वह है जो मनुष्य के भीतर है। मनुष्य का बाहरी व्यवहार उसके भीतरी व्यवहार को उजागर करता है। 


\section{व्यक्तित्व के प्रकार}

(1) अन्तर्मुखीः ऐसे व्यक्ति असामाजिक, आत्मकेन्द्रित तथा आत्म सचेत होते है। ये मिलनसार स्वाभाव के व साहसिक कार्य करने वाले नहीं होते एवं चिंतन और लेखन में रूचि रखते है ये केवल अपने आतंरिक संसार में ही व्यस्त रहते है। यही कारण है कि ऐसे व्यक्ति नेतृत्व नहीं कर पाते क्योंकि दूसरों की संगति में ये घबराहट महसूस करते हैं। आत्म-केन्द्रित रहना तथा दिन में स्वप्न देखना इनकी मुख्य विशेषता होती है।

(2) बहिर्मुखी: ऐसे व्यक्ति साहसी, मिलनसार तथा सहयोगी होते हैं तथा चिंताओं से दूर रहते हैं और उनकी वाणी में दूसरों को आकर्षित करने का अदभुत गुण होता है। ये बाहरी संसार की तरफ उन्मुख रहते है तथा दूसरों के प्रति उनका व्यवहार मित्रतापूर्ण होता है। ये सामाजिक कार्यों में रूचि लेते हैं तथा अच्छे नेता और सामाजिक कार्यकर्ता बनने का गुण विद्यमान होता है।

(3) मध्यमुखीः मध्यमुखी वे व्यक्ति होते हैं जो न पूरी तरह से अन्तर्मुखी होते हैं और न ही बहिर्मुखी। प्रत्येक व्यक्ति इन दोनों का मिश्रित रूप होता है। कुछ लोग आंशिक रूप से बहिर्मुखी होते है और कुछ आंशिक रूप से अन्तर्मुखी। कुछ अवस्थाओं में अंतर्मुखीपन और बहिर्मुखीपन समान होता है ऐसे व्यक्ति मध्यमुखी कहलाते हैं।

\section{संगीत व व्यक्तित्व}

संगीत का मनुष्य के वक्तित्व पर गहरा व सकारात्मक प्रभाव पड़ता है। संगीत एक-दूसरे के साथ सम्बन्ध स्थापित करने, अपनी भावनाओं को बाहर निकालने और मनोभावों को व्यक्त करने का सर्वोतम माध्यम है। संगीत मनुष्य के व्यक्तित्व को दिन-प्रतिदिन प्रभावित करता है तथा बिना किसी के हस्तक्षेप के सामाजिक जीवन में घुल मिल जाता है। उदाहरणार्थ आशावादी संगीत व्यक्ति के जीवन को उर्जावान बना देता है वहीं दुख भरा संगीत उसे अवसादपूर्ण बना देता है। शोधकर्ताओं ने पाया है कि जो लोग संगीत की कुछ शैलियों को पसंद करते हैं वे विशिष्ट व्यक्तित्व लक्षणों को प्रदर्शित करते हैं। मानव की दिनचर्या में प्रयोग होने वाला पसंदीदा संगीत कहीं-न-कहीं उसके व्यक्तित्व को प्रभावित करता ही है इसमें कोई संदेह नहीं है।

मनुष्य भिन्न-भिन्न प्रकार का संगीत सुनना पसंद करता है। संगीत की श्रेणी में शास्त्रीय संगीत, लोक संगीत, धार्मिक संगीत, पॉप, जैज़ इत्यादि आते हैं। लोगों के संगीत सुनने के प्रकारों से उनके व्यक्तित्व के विषय में जानकारी प्राप्त की जा सकती है। दूसरी तरफ लोगों के व्यक्तित्व के बारे में जानकर उनके संगीत सुनने के प्रकारों के बारे में भी जानकारी उपलब्ध की जा सकती है।

\section{पूर्व सम्बन्धित शोध साहित्य}

डेरी (1947) ने संगीतकारों के व्यक्तित्व गुणों और रोंमाटिक संगीत वरीयताओं के बीच सम्बन्धों से निष्कर्ष निकाला कि शारत्रीय संगीत पसन्द करने वाले संगीतकार कम भावुक और अधिक बौद्धिक 
थे जबकि रोमांटिक संगीत पंसद करने वाले लोगों ने बाहरी दुनिया में अधिक रूचि, कम भावनात्मक संयम व लोगों में अधिक रूचि दिखाई।

कैटल और एन्डरसन (1953) के अनुसार मनुष्य के मन को सन्तुष्टि प्रदान करने का संगीत एक बहुत ही बढ़िया माध्यम है तथा विभिन्न तरह के व्यक्तित्व वाले व्यक्तियों ने अलग-अलग तरह का संगीत सुनना पसन्द किया।

पायने (1967) ने पाया कि स्थिर स्वभाव वाले लोग शास्त्रीय संगीत पंसद करते हैं और विक्षिप्त स्वभाव वाले लोग रोंमाटिक संगीत पंसद करते है। पायने ने व्यक्तित्व के र्थिर व विक्षिप्त आयाम तथा संगीत में शास्त्रीय व रोंमाटिक संगीत के बीच एक सकारात्मक सम्बन्ध बताया।

शोध प्रविधि

उद्देश्य

- विद्यार्थियों का व्यक्तित्व तथा संगीत सुनने की रूचि का अध्ययन

क्षेत्र

इस शोध कार्य के अन्तर्गत हिमाचल प्रदेश विश्वविद्यालय, शिमला के स्नातकोत्तर विद्यार्थियों के व्यक्तित्व गुणों के आधार पर उनकी संगीत सुनने की रूचि के अध्ययन पर केन्द्रित रहा।

शोध विधि

शोध कार्य के लिए सर्वेक्षण विधि का प्रयोग किया गया।

न्यायदर्श

हिमाचल प्रदेश विश्वविद्यालय के विभिन्न विभागों में अध्ययनरत 120 स्नातकोत्तर विद्यार्थियों को यादृच्छिक प्रतिचयन पद्धति द्वारा शोध कार्य के लिए लिया गया जिनमें से 20 विद्यार्थियों ने प्रश्नावली को स्पष्ट रूप ने नहीं भरा अतः 120 विद्यार्थियों में से 100 विद्यार्थियों को ही चुना गया जिन्होंने प्रश्नावली को स्पष्ट रूप से भरा जिनमें 50 छात्र व 50 छात्राएँ सम्मिलित किए गए।

तालिका 1: हिमाचल प्रदेश विश्वविद्यालय के विभिन्न विभागों से सम्बन्धित स्नातकोत्तर विद्यार्थी

\begin{tabular}{|ccc|}
\hline क्र.सं. & विद्यार्थी & विद्यार्थियों की संख्या \\
\hline 1. & छात्र & 50 \\
\hline 2. & छात्राएँ & 50 \\
\hline & कुल & 100 \\
\hline
\end{tabular}


हिमाचल प्रदेश विश्वविद्यालय के स्नातकोत्तर के 100 विद्यार्थियों में से क्रमश : अन्तर्मुखी व्यक्तित्व के 49 विद्यार्थी व बहिर्मुखी व्यक्तित्व के 51 विद्यार्थी थे।

तालिका 2: हिमाचल प्रदेश विश्वविद्यालय के अन्तर्मुखी व बहिर्मुखी व्यक्तित्व के विद्यार्थी

\begin{tabular}{|ccc|}
\hline क्र.सं. & अन्तर्मुखी / बहिर्मुखी & विद्यार्थियों की संख्या \\
\hline 1. & अन्तर्मुखी & 49 \\
\hline 2. & बहिर्मुखी & 51 \\
\hline & कुल & 100 \\
\hline
\end{tabular}

उपकरण

शोध सम्बन्धित सामग्री को प्राप्त करने के लिए Eysenck की Personality Inventory (EPI) (Extroversion/Introversion) प्रश्नावली का प्रयोग किया गया तथा व्यक्तिगत जानकारी सूची का निर्माण व प्रयोग किया गया।

\section{शोध प्रक्रिया}

शोधकर्ताओं ने हिमाचल प्रदेश विश्वविद्यालय के विभिन्न विभागों में जाकर स्नातकोत्तर में अध्ययनरत 120 विद्यार्थियों से व्यक्तिगत जानकारी सूची व Personality Inventory (EPI) प्रश्नावली भरवाई गई जिसमें छात्र व छात्राएं दोनों सम्मिलित थे। प्रश्नावली भरवाने के बाद यह पाया गया कि उनमें से कुछ विद्यार्थियों ने प्रश्नों को उचित ढंग से नहीं पढ़ा व अधूरा भरा तथा कुछ विद्यार्थियों की व्यक्तिगत जानकारी में त्रुटि पाई गई। 120 विद्यार्थियों में से केवल 100 विद्यार्थियों ने प्रश्नावली को पूर्ण रूप से भरा व 20 विद्यार्थियों ने प्रश्नावली को पूर्ण रूप से नहीं भरा। परिणाम स्वरूप 100 विद्यार्थियों को ही शोध में सम्मिलित किया गया। इसके पश्चात् प्राप्त आंकड़ों का विश्लेषण किया गया।

विश्लेषण एवं व्याख्या

हिमाचल प्रदेश विश्वविद्यालय के स्नातकोत्तर विद्यार्थियों की सांगीतिक रूचियों का अध्ययन

हिमाचल प्रदेश विश्वविद्यालय के स्नातकोत्तर विद्यार्थियों की सांगीतिक रूचि तथा वरीयता को तालिका 3 से दर्शाया गया है। 
तालिका 3

\begin{tabular}{|cccc|}
\hline क्र.सं. & सांगीतिक विधा & विद्यार्थियों की संख्या & वरीयता \\
\hline 1. & शास्त्रीय संगीत & 13 & 6 \\
\hline 2. & गज़ल & 18 & 1 \\
\hline 3. & लोक संगीत & 16 & 3 \\
\hline 4. & भजन & 15 & 4 \\
\hline 5. & ठुमरी & 2 & 8 \\
\hline 6. & कव्वाली & 5 & 7 \\
\hline 7. & पाश्चात्य & 14 & 5 \\
\hline 8. & फिल्मी & 17 & 2 \\
\hline
\end{tabular}

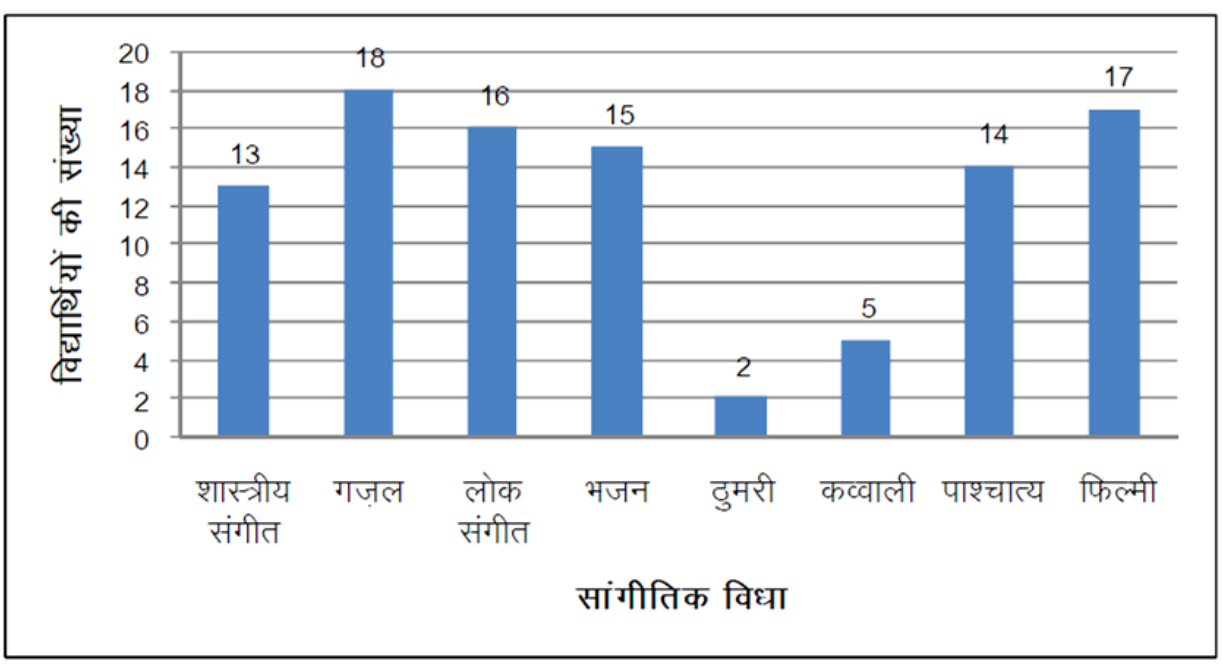

ग्राफ 1

तालिका 3 से ज्ञात होता है कि हिमाचल प्रदेश विश्वविद्यालय के विभिन्न विषयों से सम्बन्धित 100 स्नातकोत्तर विद्यार्थियों में से 18 विद्यार्थियों ने गज़ल विधा को सर्वाधिक पंसद किया दूसरे स्थान पर 17 विद्यार्थियों ने फिल्मी संगीत को पंसद किया, तीसरे स्थान पर 16 विद्यार्थियों ने लोक संगीत को पंसद किया चौथे स्थान पर 15 विद्यार्थियों ने भजन संगीत को पंसद किया। पांचवे स्थान पर 14 विद्याार्थियों ने पाश्चात्य संगीत पंसद किया छठे स्थान पर 13 विद्यार्थियों ने शास्त्रीय संगीत को पंसद किया सातवें स्थान पर 5 विद्यार्थियों ने कव्वाली को पसंद किया व आंठवे स्थान पर 2 विद्यार्थियों ने ठुमरी विधा को पंसद किया।

हिमाचल प्रदेश विश्वविद्यालय के अन्तर्मुखी व्यक्तित्व के विद्यार्थियों की सांगीतिक रूचि का अध्ययन

हिमाचल प्रदेश विश्वविद्यालय के अन्तर्मुखी व्यक्तित्व के विद्यार्थियों की सांगीतिक रूचि तथा वरीयता को तालिका 4 से दर्शाया गया है। 


\section{तालिका 4}

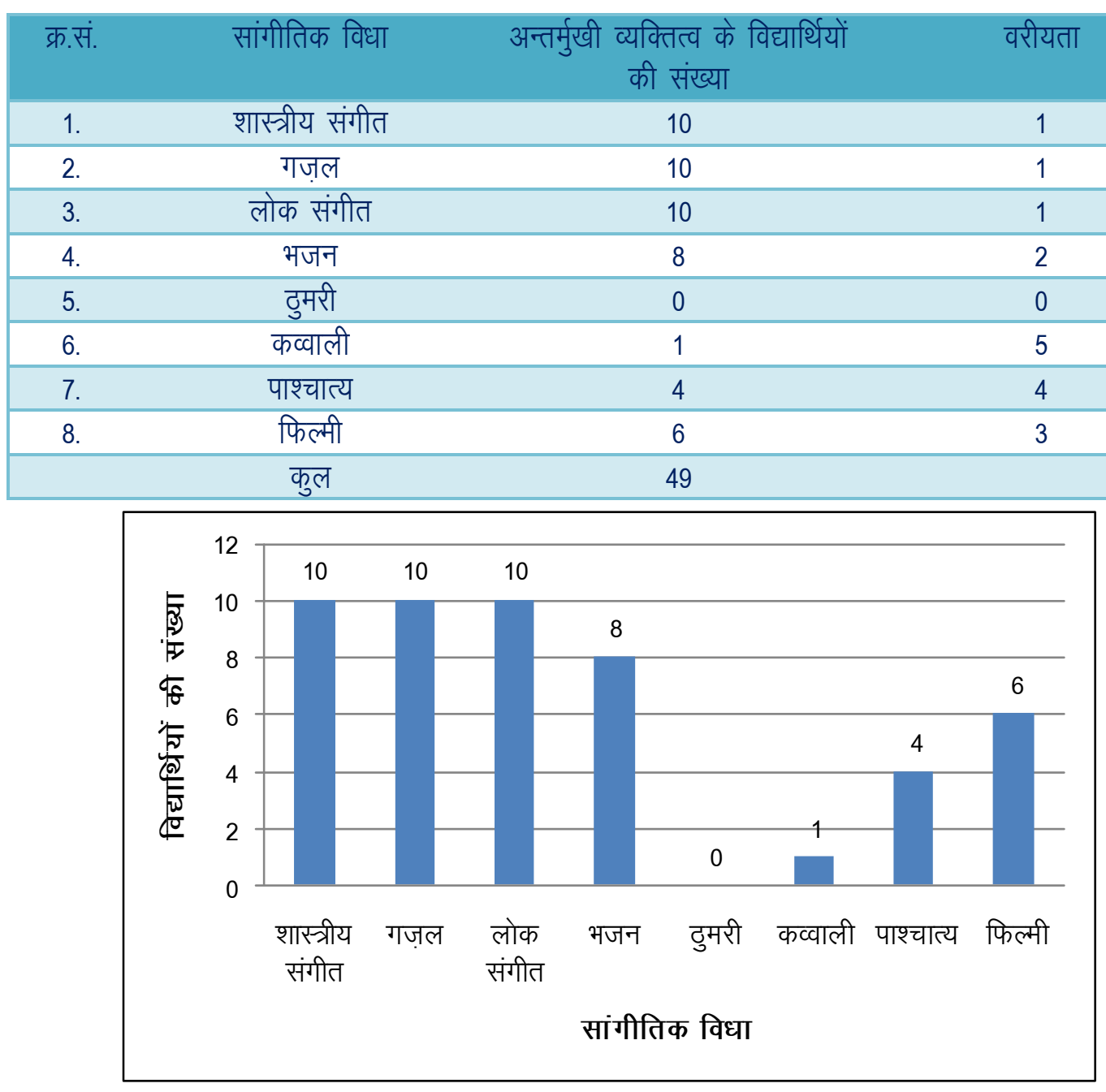

ग्राफ 2

तालिका 4 से यह ज्ञात होता है कि हिमाचल प्रदेश विश्वविद्यालय के अन्तर्मुखी व्यक्तित्व के कुल 49 विद्यार्थियों में से 10 विद्यार्थियों ने क्रमशः शास्त्रीय संगीत, गज़ल व लोक संगीत को प्रथम स्थान पर चुना। दूसरे स्थान पर 8 विद्यार्थियों ने भजन विधा को चुना। तीसरे स्थान पर 6 विद्यार्थियों ने फिल्मी विधा को चुना। चौथे स्थान पर 4 विद्यार्थियों ने पाश्चात्य संगीत को चुना। पाँचवें स्थान पर केवल 1 विद्यार्थी ने कव्वाली विधा को चुना व ठुमरी विधा में किसी भी विद्यार्थी ने अपनी रूचि नहीं दिखाई।

हिमाचल प्रदेश विश्वविद्यालय के बहिर्मुखी व्यक्तित्व के विद्यार्थियों की सांगीतिक रूचि का अध्ययन हिमाचल प्रदेश विश्वविद्यालय के बहिर्मुखी व्यक्तित्व के विद्यार्थियों की सांगीतिक रूचि तथा वरीयता को तालिका 5 से दर्शाया गया है। 
तालिका 5

\begin{tabular}{|cccc|}
\hline क्र.सं. & सांगीतिक विधा & बहिर्मुखी व्यक्तित्व के विद्यार्थियों की संख्या & वरीयता \\
\hline 1. & शास्त्रीय संगीत & 3 & 7 \\
\hline 2. & गज़ल & 8 & 3 \\
\hline 3. & लोक संगीत & 6 & 5 \\
\hline 4. & भजन & 7 & 4 \\
\hline 5. & ठुमरी & 2 & 8 \\
\hline 6. & कव्वाली & 4 & 6 \\
\hline 7. & पाश्चात्य & 10 & 2 \\
\hline 8. & फिल्मी & 11 & 1 \\
\hline & कुल & 51 & \\
\hline
\end{tabular}

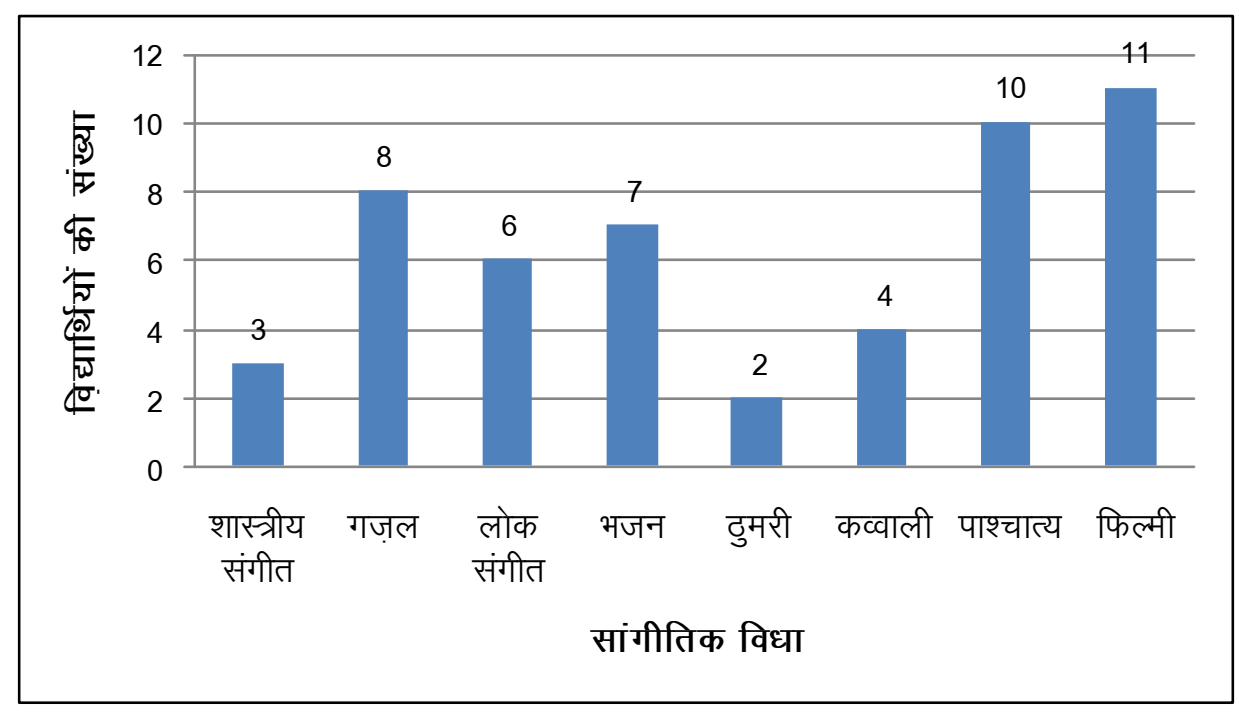

ग्राफ 3

तालिका 5 से यह ज्ञात होता है कि हिमाचल प्रदेश विश्वविद्यालय के बहिर्मुखी व्यक्तित्व के कुल 51 विद्यार्थियों में से 11 विद्यार्थियों ने फिल्मी विधा को सर्वाधिक पसन्द कर प्रथम स्थान पर चुना। 10 विद्यार्थियों ने पाश्चात्य संगीत को दूसरे स्थान पर चुना। 8 विद्यार्थियों ने गज़ल विधा को तीसरे स्थान पर चुना। 7 विद्यार्थियों ने भजन संगीत को चौथे स्थान पर चुना। 6 विद्यार्थियों ने लोक संगीत विधा को पाँचवे स्थान पर चुना। 4 विद्यार्थियों ने कव्वाली विधा को छठे स्थान पर चुना। 3 विद्यार्थियों ने शास्त्रीय संगीत को सांतवें स्थान पर चुना व ठुमरी विधा को 2 विद्यार्थियों ने आठवें स्थान पर चुना। 


\section{परिणामों की परिचर्चा एवं निष्कर्ष}

हिमाचल प्रदेश विश्वविद्यालय में अध्ययनरत छात्र तथा छात्राओं नें गज़ल विधा को अपनी प्रथम सांगीतिक रूचि के रूप में चुना। दूसरे स्थान पर इन्हीं विद्यार्थियों नें फिल्मी विधा को सांगीतिक रूचि के रूप में चुना। तीसरे, चौथे, पाँचवे, छठे, सातवें व आठवें स्थान पर विद्यार्थियों नें क्रमशः लोक संगीत, भजन, पाश्चात्य, शास्त्रीय संगीत, कव्वाली व ठुमरी विधा को चुना। इन सभी सांगीतिक विधाओं के प्रति रुचि तथा आकर्षण के कारण विद्यार्थी की स्वयं की रूचि तथा पारिवारिक वातावरण होता है। कई विद्यार्थियों में किसी विशेष विधा की तरफ आकर्षण तथा लगाव वांशिक रूप में भी होता है। इन सभी सांगीतिक विधाओं में विद्यार्थियों नें शास्त्रीय संगीत को छठे स्थान पर सांगीतिक रूचि के रूप में स्वीकार किया। इन सांगीतिक विधाओं की तरफ विद्यार्थियों की रूचि गज़ल, फिल्मी, लोक संगीत, भजन, पाश्चात्य संगीत की अपेक्षा कम है। इसका कारण शास्त्रीय संगीत के प्रति समाज की संकुचित सोच तथा रूढ़िवादी धारणा हो सकती है। शास्त्रीय संगीत अन्य सांगीतिक विधाओं की तरह विद्यार्थियों में अधिक प्रचलित नहीं है, इसका कारण शास्त्रीय संगीत के प्रचार-प्रसार में कमी, शास्त्रीय संगीत के प्रति समाज में जागरूकता की कमी हो सकती है।

शोध में पाया गया कि अंतर्मुखी व्यक्तित्व के विद्यर्थियों ने शास्त्रीय संगीत, लोक संगीत व गज़ल विधा को सर्वाधिक पसंद किया। इसका कारण यह हो सकता है कि अंतर्मुखी व्यक्तित्व के लोग शांत, विश्वसनीय, धीर-गंभीर होते हैं तथा शास्त्रीय संगीत व गज़ल जैसी विधाएं गंभीर व मन को सुकून देने वाली विधाएं हैं, वहीं बहिर्मुखी व्यक्तित्व के विद्यार्थियों नें फिल्मी संगीत विधा को प्रथम स्थान पर व पाश्चात्य संगीत विधा को द्वितीय स्थान पर चुना। इसका कारण यह हो सकता है कि बहिर्मुखी व्यक्तित्व के लोग बातुनी, चंचल व मिलनसार प्रकृति के होते हैं तथा पाश्चात्य संगीत विधा व फिल्मी विधाएं आदि चंचल प्रकृति की विधाएं हैं।

\section{उपसंहार}

इन सभी तथ्यों से यह निष्कर्ष निकाला जा सकता है कि

- हिमाचल प्रदेश विश्वविद्यालय के स्नातकोतर में अध्ययनरत विद्यार्थी ग़ज़ल को सर्वाधिक पसंद करते हैं, दूसरे स्थान पर फिल्मी संगीत विधा को पसंद करते हैं। तीसरे, चौथे, पाँचवे, छठे, सातवें व आठवें स्थान पर विद्यार्थियों नें कमशः लोक संगीत, भजन, पाश्चात्य, शास्त्रीय संगीत, कव्वाली व ठुमरी विधा को पसंद करते हैं।

- हिमाचल प्रदेश विश्वविद्यालय के स्नातकोतर में अध्ययनरत अंतर्मुखी व्यक्तित्व के विद्यार्थी शास्त्रीय संगीत, ग़ज़ व लोक संगीत सर्वाधिक पसंद करते हैं तथा दूसरे तीसरे, चौथे व पाँचवे स्थान पर भजन, फिल्मी, पाश्चात्य व कव्वाली को पसंद करते है। 
- हिमाचल प्रदेश विश्वविद्यालय के स्नातकोतर में अध्ययनरत बहिर्मुखी व्यक्तित्व के विद्यार्थी फिल्मी विधा को सर्वाधिक पसंद करते हैं, दूसरे स्थान पर पाश्चात्य संगीत विधा को पसंद करते हैं तथा तीसरे, चौथे, पाँचवे, छठे, सातवें व आठवें स्थान पर क्रमशः गज़ल, भजन, लोक संगीत, कव्वाली, शास्त्रीय संगीत व ठुमरी को पसंद करते हैं।

सन्दर्भ ग्रन्थ

तिवारी, डॉ. किरण. (2015). संगीत एवं मनोविज्ञान, कनिष्क पब्लिशर्स एंड डिस्ट्रीब्यूटर्स, नई दिल्ली।

शर्मा, डॉ. मृत्युंजय. (2001). संगीत मैनुअल, एच.जी. पब्लिकेशन्स, नई दिल्ली।

Cattell, R. B.\& Andorson, J.D.(1953). The measurement of personality and behaviour disorders by the I.P.A.T. music preference test, Journal of applied psychology, 77 (6)446454.

Cattell, R.B. and Saunders, D.R. (1954) Musical preferences and personality diagnosis: A factorization of one hundred and twenty themes. Journal of social psychology. 39, 3-24.

Deri, O. (1947). Musical taste and personality. Unpublished Master's thesis, Columbia University Teachers College, New York.

Mayeske, G.W. (1962). Some aspects of musical preference dimensions of personality. Dissertation Abstracts, 23, 346 8B. University Microfilms No. 62- 06, 188.

Payne, E (1967). Musical taste and personality. British Journal of Psychology, 58. 133-138. 